\title{
Changes in Posture Control of Women That Fall During Pregnancy
}

\author{
Kaname Takeda ${ }^{1^{*} \mathbb{D}}$, Hiromi Yoshikata ${ }^{2}$, Masumi Imura ${ }^{3}$
}

\begin{abstract}
Objectives: A survey of falls during pregnancy conducted in the United States and Japan found that the frequency of falls increased with the progression of pregnancy. The purpose of this study was to analyze the posture control properties of women that fall during pregnancy.

Materials and Methods: Participants comprised 100 pregnant women (age, 20-30 years). Posture control was assessed during the second and third trimesters using 2 stabilometers to measure the ability to control upright posture. During the third trimester, participants were asked to complete a self-administered questionnaire on falls.

Results: Data were obtained from 82 subjects after excluding dropouts. Ten fallers were identified, resulting in a fall incidence of $12 \%$. The fall group showed a $9.9 \%$ increase in abdominal girth from the second to third trimester, significantly greater than the $6.9 \%$ in the non-fall group. In the fall group, the rectangular area in the back, left and right was larger from the second trimester. In the third trimester, the stability limits of the fall group were significantly smaller than in the non-fall group.

Conclusions: The loosening of joints from second trimester and declines in equilibrium function and an abrupt increase in abdominal girth may cause shifts in recognition of how the body moves, such as estimation errors, thereby causing falls. For pregnant women, guidance, exercises promoting awareness of fall prevention, and accurate perception of the body need to be incorporated in the exercise from the second trimester.

Keywords: Pregnant women, Posture control, Estimation error
\end{abstract}

\section{Introduction}

According to a survey in the United States, about 26\% of working pregnant women have experienced falls, a rate comparable to falls among elderly people $\geq 65$ years old (1). Major falls account for $17 \%-39 \%$ of maternal traumas requiring treatment, resulting in fetal death in 3\%-7\% of these falls (2). Fall prevention is thus an important issue for pregnant women. In a survey conducted by Takeda et al targeting 1000 pregnant women in urban areas of Japan, 19\% had experienced falls (3). Prevention of falls requires posture control, which includes elements such as bone alignment, joint function, and muscular strength, in addition to neural mechanisms related to equilibrium. Several factors that reduce posture control ability have been identified in pregnancy, including increased spinal lordosis, reduced strength of the abdominal muscles, and loosening of the major joints (4-6). Fluctuations in the center of gravity during pregnancy are reported to increase postural sway fluctuations in the anteroposterior and radial directions (7). Butler et al reported increased postural sway throughout pregnancy, as evidenced by the increased length of the center of pressure during quiet stance in pregnant women (8). Takeda et al reported that maximum center of pressure displacement in the standing position was reduced in the second and third trimesters, while the maximum mass transfer rate in the forward direction, including diagonal directions, was decreased in the third trimester (9). Stability of posture can be regarded as the ability to keep the center of gravity of the body within the range of the stability limit (10). A fall thus represents a state in which deviation of the center of gravity of the body from the stability limit cannot be restored. A situation in which the center of gravity of the body shows an increased likelihood of deviating from the stability limit is unstable because posture stability cannot be maintained even in the event of small external forces. The increase in the number of falls accompanying progression of pregnancy thus seems to be due to the fact that the center of gravity of the body deviates from the stability limit with even a small external force because of physical changes.

In a survey of fall situations targeting 2000 pregnant women in Japan, Takeda et al reported that falls due to wobble during anti-weight/gravity actions such as descending stairs, squatting/rising when sitting increased with pregnancy (3). The rates of increase in abdomen,

Received 14 September 2017, Accepted 20 January 2018, Available online 5 February 2018

${ }^{1}$ Division of Physical Therapy, Department of Rehabilitation Sciences, Faculty of Allied Health Sciences, Kansai University of Welfare Sciences, 3-11-1 Asahigaoka, Kashiwara City, Osaka 582-0026, Japan. ${ }^{2}$ Yoshikata Obstetrics and Gynecology, 2430 Kozukuecho, Kohoku ward, Yokohama City, Kanagawa 222-0036, Japan. ${ }^{3}$ Japanese Red Cross College of Nursing, Graduate School of Nursing, International Health Care and Midwifery, 4-1-3 Hiroo, Shibuya-ku, Tokyo 150-0012, Japan.

*Corresponding Author: Kaname Takeda, Tel: +81729780088, Email: takeda@tamateyama.ac.jp 
chest, and body weight are marked at 20-24 weeks of pregnancy and 32-36 weeks of pregnancy (11). In the third trimester, the weight gain of the mother is approximately 10-12 kg (12), further increasing the burden on the musculoskeletal system. "Wandering" indicates a situation in which relative muscular strength is insufficient to support the weight gain inherent during the course of pregnancy.

Many studies have investigated changes in posture control during pregnancy, but none have examined the characteristics of fallers from the perspective of posture control.

\section{Objectives}

In considering fall prevention, understanding the characteristics of posture control, including the position of the center of gravity, weight, and physical characteristics including abdominal girth, may help to support fall prevention in daily life during pregnancy. The purpose of this study was to clarify the posture control characteristics of women that fall during pregnancy. The significance of this research lies in the provision of basic quantitative data on factors contributing to falls during pregnancy, thus laying the groundwork for future examination of interventions, including fall guidance.

\section{Materials and Methods}

Subjects were 100 healthy pregnant women in their 20's and 30's who understood the purpose and content of the study and had consulted the Obstetrics and Gynecology Department, where they provided consent to participate. Exclusion criteria included 1) a history of lower limb or lower back injury; or 2) regularly (at least once per week) participating in sports. Measurements were taken at the Obstetrics and Gynecology Measurement Office, where obstetricians, gynecologists and midwives are on call in case there are unforeseen circumstances involving pregnant women and fetuses. After the women's physical condition was checked during regular health checks by gynecologists, measurements were carried out. These measurements were carried out in the second and third trimesters to assess changes over time. For quantitative evaluation of posture control ability, 2 stabilometers (JK101 II; Unimec Co., Tokyo) and a personal computer were used (Figure 1a). As a measurement task, each subject was instructed to maintain a stationary standing position on the 2 stabilometers while barefoot, with the medial malleoli $100 \mathrm{~mm}$ apart.

The subject was instructed to signal "yes" when body sway was considered stable and to then maintain the same posture for another $10 \mathrm{~s}$. The subject was then asked to maintain the stable posture while the center of gravity was moved as far as possible in the forward direction, in the same manner as in static standing. Thereafter, the center of gravity was moved backward in the same manner, then to the right, then to the left. In each case, data were extracted for 10 seconds from the time the subject signaled to start the center of gravity fluctuation in each position. These tasks were conducted twice during each measurement session. Analyzed parameters were as follows (Figure 1b): 1) rectangular area(the area in which the body sways) for the posture held for 10 seconds at the front, back, left and right positions in the second and third trimesters; 2) center-of-gravity movement for each of the front,back, right and left (anterioposterio center-of-gravity movement was calculated by determining the middle point of each rectangular area when holding the posture for 10 seconds to the front or back; lateral center-of-gravity movement was calculated using the same process); and 3) stability limits, obtained by multiplying the movement of the center of gravity between the anterioposterio and lateral as obtained in 2). Physical characteristics were determined by interviews conducted before the posture control measurements. These interviews were based on the results of periodic medical examinations in the second or third trimester. The items in the questionnaire created for this

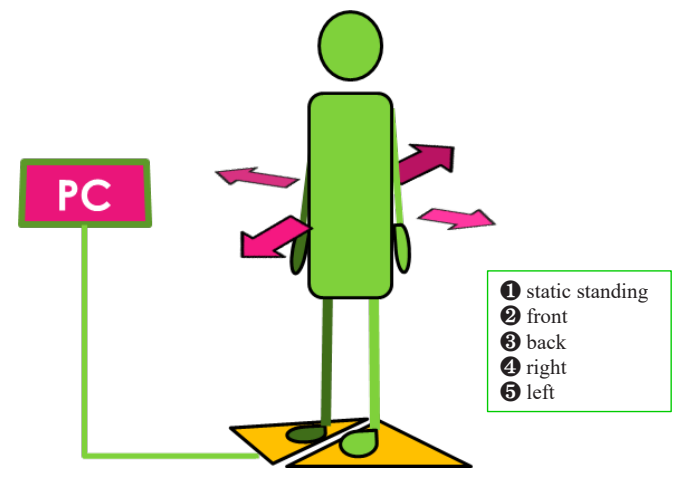

The amount of instability sway of the center of gravity at each position was extracted from 10 seconds of data once the woman's motion subsided at the new position.

a) Stabilometer system and task

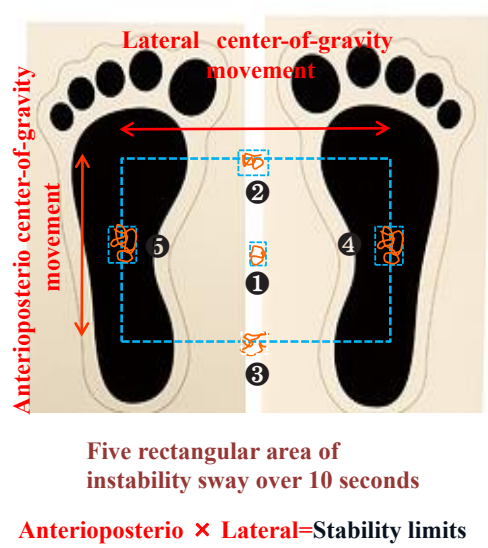

b)Parameters

Figure 1. Stabilometer System and Task and Parameters. 
study were height, weight and abdominal circumference. To investigate the situation of falls during pregnancy, a self-administered questionnaire was provided in the third trimester and collected in the maternity hospital after birth. The survey questionnaire collected information on the presence or absence of falls during pregnancy, frequency, environment, time zone, and use of medications. The definition of fall in this study was as described by Gibson et al, "unintentionally coming to the ground or some lower level and other than as a consequence of sustaining a violent blow, loss of consciousness, sudden onset of paralysis as in stroke or an epileptic seizure" (13). Measurements were carried out with the approval of the ethics committee at Kansai University of Welfare Science (approval number 14-10). For statistical processing, SPSS Statistics version 24 (IBM, Japan) was used for: 1) comparison of age and height between subjects in the nonfall and fall group, and 2) comparison of posture control parameters and 2 factors: point in time during pregnancy and fall experience. Wilcoxon's signed rank sum test was used to analyze 1) The verification of 2) was verified by Two-way repeated measures analysis of variance and for subsequent verification Bonferroni correction was used $(P<0.05)$.

\section{Results}

Subjects comprised 82 women (mean age, $32 \pm 3.2$ years; height, $158.2 \pm 5.3 \mathrm{~cm}$ ) after excluding participants who dropped out. Reasons for dropping out included an inability to participate in the second measurement day due to poor physical condition or traffic congestion. Ten of the 82 participants experienced falls, representing a fall incidence of $12 \%$. Characteristics of the 10 fallers are shown in Table 1. The mean abdominal circumference was $92.2 \pm 3.3 \mathrm{~cm}$. Four women fell in the second trimester and 6 women fell in the third trimester. In the second trimester, falls most often occurred while walking, and a case of slipping and falling and a case of twisting the ankle were confirmed. In the third trimester, many falls occurred during squatting and rising motions, and these falls resulted from wobbling and wandering. Regarding medication, two fallers were taking medicine for constipation, one was taking medicine for anemia, and one was taking cough suppressants.

The average age and average height of subjects in the non-fall group were 32 years and $158.4 \mathrm{~cm}$, respectively, and there were no significant differences from the fall group. Table 2 shows body mass index (BMI), body weight, abdominal circumference and rate of change in the non-fall and fall group. There was no significant difference in the interaction for BMI $(F(1,162)=0.831$, $P>0.05)$, but a significant difference was observed in the main effect of time $(\mathrm{F}[1,162]=417.99, P<0.05)$. BMI in each group increased significantly with the progression of pregnancy $(P=0.01)$, by $5.8 \%$ in the non-fall group and $6.3 \%$ in the fall group. There was no significant difference in the interaction for body weight $(\mathrm{F}[1,162]=0.215$, $P>0.05)$, but a significant difference was observed in the main effect of time ( $F[1,162]=432.07, P<0.05)$. In each group, body weight significantly increased $(P=0.01)$ with the progression of pregnancy, by $6.1 \%$ in the non-fall group and $6.4 \%$ in the fall group. There was a significant difference in the interaction for abdominal circumference $(\mathrm{F}[1,162]=10.27, P<0.05)$, the main effect of time $(\mathrm{F}[1,162]=375.38, P<0.05)$ and the main effect of experiencing a fall $(\mathrm{F}[1,162]=4.123, P<0.05)$.In the subsequent simple main effect test, abdominal girth was significantly larger in the non-fall group in the second trimester $(P=0.01)$, but there was no significant difference between the groups at the end of pregnancy $(P=0.34)$.In each group, abdominal girth increased significantly with the progression of pregnancy, but the rate of increase in abdominal girth from the second to the third trimester increased by $9.9 \%$ in the fall group, compared to $6.9 \%$ in the non-fall group, representing a significant increase.

Table 3 shows changes in posture control ability with progression of pregnancy with or without falls. In the rectangular area in each direction, there was no interaction in both positions that the static standing position ( $F$ $(1,162)=0.000, P>0.05)$ and the front direction $(\mathrm{F}(1,162)$ $=0.299, P>0.05)$. There was no significant difference in the main effect, respectively, the time and experiencing a fall. There was also no significant difference in the main effect.

There was no significant difference in interaction in the back rectangular area $(\mathrm{F}(1,162)=0.575, P>0.05)$, and the only main effect was the experiencing a fall $(\mathrm{F}(1,162)$ $=8.470, P<0.05)$. Compared to the non-fall group, back rectangular area was significantly larger in the fall group at every time point $(P=0.01)$. There were no significant differences in the interaction in the right rectangular area (F $(1,162)=1.508, P>0.05)$, the main effect of time ( $F$ $(1,162)=5.60, P<0.05)$, or the main effect of experiencing a fall $(\mathrm{F}[1,162]=8.036, P<0.05)$. Compared to the non-fall group, the fall group was significantly larger at every time point $(P=0.01)$, and the rectangular area in each group was significantly increased to $8.1 \%$ (non-fall group) and $21.3 \%$ (fall group $)(P=0.02)$, respectively, with the progression of pregnancy. There were no significant differences in the interaction in the left rectangular area $(\mathrm{F}$ $[1,162]=1.535, P>0.05)$, the main effect of time $(\mathrm{F}(1,162)$ $=5.78, P<0.05)$, or the main effect of experiencing a fall $(\mathrm{F}[1,162]=13.63, P<0.05)$. Compared with the non-fall group, the fall group was significantly larger $(P=0.01)$ at each time point, and the rectangular area significantly increased to $10.7 \%$ (non-fall group) and $25.1 \%$ (fall group) in each group with the progression of pregnancy $(P=0.01)$.

A significant interaction was observed between the anteroposterior center-of-gravity movement (F $[1,162]=5.438, \quad P<0.05)$, and a main effect of time was observed $(\mathrm{F}[1,162]=13.01, \mathrm{P}<0.05)$. There was no 


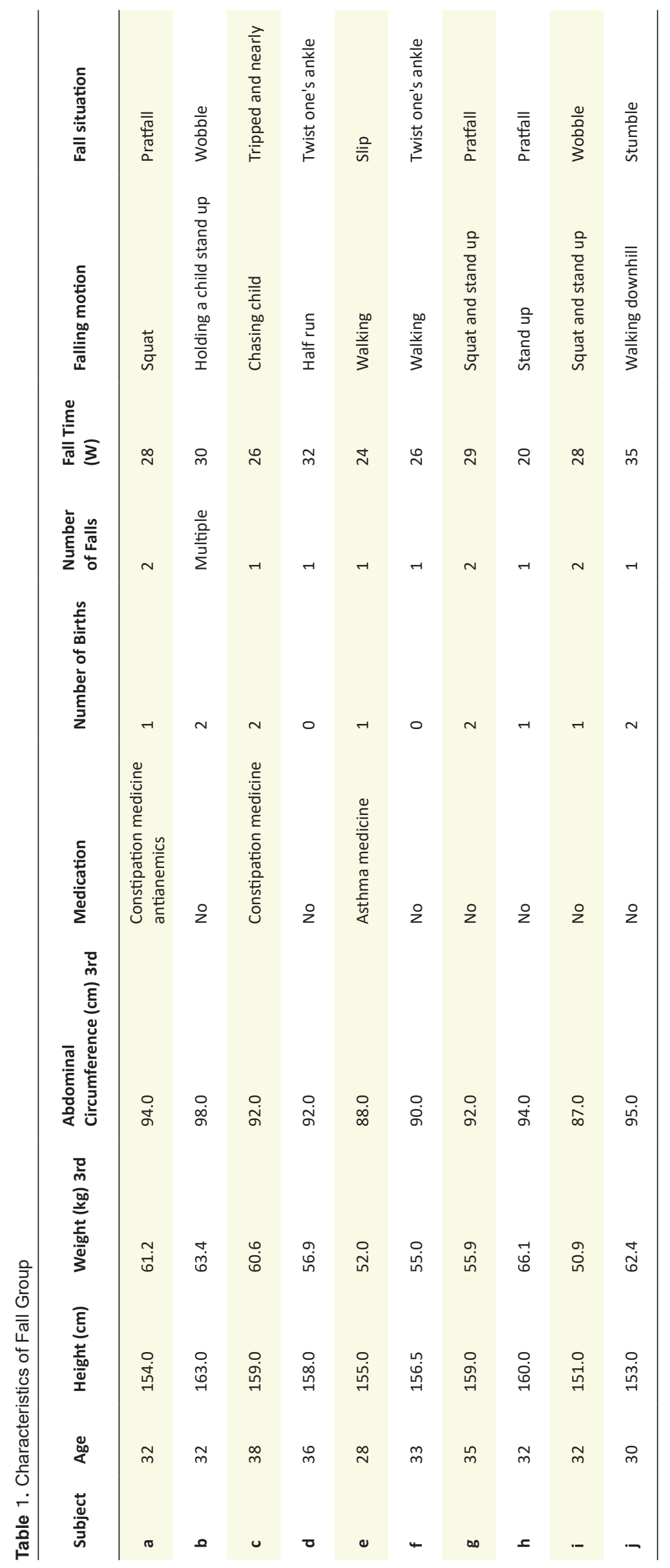


Table 2. Comparison Between Time and Experiencing a Fall About BMI, Body Weight and Abdominal Circumference.

\begin{tabular}{|c|c|c|c|c|c|c|c|c|c|c|c|}
\hline & & \multicolumn{3}{|c|}{ 2nd trimester } & \multicolumn{3}{|c|}{ 3rd Trimester } & $\begin{array}{l}\text { Change } \\
\text { rate (\%) }\end{array}$ & $\begin{array}{l}\text { Time Main } \\
\text { Effect }\end{array}$ & $\begin{array}{l}\text { Experiencing a } \\
\text { Fall Main Effect }\end{array}$ & Interaction \\
\hline \multirow[t]{2}{*}{ BMI } & Non-fall group & \multicolumn{3}{|l|}{22.4} & \multicolumn{3}{|l|}{23.7} & 5.8 & \multirow[t]{2}{*}{ 417.99* } & \multirow[t]{2}{*}{0.83} & \multirow[t]{2}{*}{0.83} \\
\hline & Fall group & \multicolumn{3}{|l|}{22.3} & \multicolumn{3}{|l|}{23.7} & 6.3 & & & \\
\hline \multirow[t]{2}{*}{ Body weight $(\mathrm{kg})$} & Non-fall group & 56.1 & \pm & 6.4 & 59.5 & \pm & 6.5 & 6.1 & \multirow[t]{2}{*}{$432.07^{*}$} & \multirow[t]{2}{*}{0.60} & \multirow[t]{2}{*}{0.22} \\
\hline & Fall group & 54.9 & \pm & 4.9 & 58.4 & \pm & 4.9 & 6.4 & & & \\
\hline \multirow{2}{*}{$\begin{array}{l}\text { Abdominal } \\
\text { circumference }(\mathrm{cm})\end{array}$} & Non-fall group & 87.3 & \pm & 5.2 & 93.3 & \pm & 4.8 & 6.9 & \multirow[t]{2}{*}{$375.38^{*}$} & \multirow[t]{2}{*}{$4.123^{*}$} & \multirow[t]{2}{*}{$10.27^{*}$} \\
\hline & Fall group & 83.9 & \pm & 5.2 & 92.2 & \pm & 3.2 & 9.9 & & & \\
\hline
\end{tabular}

Non-fall group( $n=72)$, Fall group $(n=10), * P<0.05$.

significant difference between the groups in the second trimester $(P=0.07)$ in the subsequent simple main effect test, but in the third trimester, the fall group was significantly smaller $(P=0.01)$. There was no change $(P=0.90)$ with pregnancy in the non-fall group, and the anteroposterior center-of-gravity movement was significantly decreased by $8.7 \%$ in the fall group $(P=0.04)$. A significant interaction was observed with the lateral center-of-gravity movement $(\mathrm{F}(1,162)=9.867, P<0.05)$, the main effect of time $(\mathrm{F}[1,162]=6.189, P<0.05)$, and the main effect of experiencing a fall $(\mathrm{F}(1,162)=8.451$, $P<0.05)$. Although there was no significant difference between the groups in the second trimester $(P=0.35)$ in the subsequent simple main effect test, the lateral center-of-gravity movement was significantly smaller in the fall group in the third trimester $(P=0.01)$. There was no change $(P=0.17)$ with pregnancy in the non-fall group, and the lateral center-of-gravity movement was significantly decreased by $6.2 \%$ in the fall group $(P=0.01)$. There was a significant interaction in the stability limits $(\mathrm{F}(1,162)=9.086, P<0.05)$, the main effect of time ( $\mathrm{F}$ $(1,162)=13.19, P<0.05)$, and the main effect of fall $(\mathrm{F}$ $(1,162)=5.037, P<0.05)$.In the subsequent simple main effect test, although there was no significant difference between the groups in the second trimester $(P=0.38)$, the stability limits was significantly smaller in the fall group on the third trimester $(P=0.01)$. There was no change $(P=0.39)$ with pregnancy in the non-fall group, and the stability limits were significantly decreased by $13.7 \%$ in the falling group $(P=0.01)$.

\section{Discussion}

Greater stability of posture maintenance is achieved with a lower center of gravity, and also when there is a low probability that the center of gravity line will deviate from the stable region (14). In the present research, based on the idea advocated by Mochizuki, the rectangular area at the time of posture holding at the position of center of gravity movement was arbitrarily considered as posture-holding ability in each direction (14). The change in posture control during pregnancy was examined by capturing the stability limits calculated anteroposterior and lateral center-of-gravity movement and stability limits.

A characteristic of the posture control in the fall group was that the anteroposterior center of gravity movement decreased significantly as pregnancy progressed. Normally, ankle joint flexors are used in what is called an ankle strategy to control anteroposterior posture while maintaining the base of support, but when posture control through the ankle strategy becomes insufficient, a hip strategy is also used for posture control with hip muscles (10). Subjects in the present study were required to move their center of gravity to the anteroposterior, lateral as much as possible during measurements. Therefore, they needed to employ a hip joint strategy during the final range of movement of the center of gravity. In the static upright position during pregnancy, balance with the center of gravity of the body displaced forward and upward is maintained by anterior positioning of the center of gravity line $(15,16)$. Abdominal circumference significantly increased in the third trimester. Thus, we assumed that the physical center of gravity in the third trimester had shifted forward, and that the range of motion of the hip joint was restricted due to the forward tilt of the pelvis. For these reasons, the amount of forward movement of the center of gravity was considered decreased in the third trimester, and the amount of anteroposterior movement of the center of gravity was decreased.

Subjects in the fall group exhibited smaller movement of the center of gravity in both the anteroposterior and lateral directions. Factors considered important in posture control include stabilization of the sacroiliac joint via the abdominal oblique muscle during lateral weight shift, retention of the horizontal position of the pelvis via the gluteus muscles, and maintaining the vertical position of the trunk (17). Subjects in the fall group exhibited a sharp increase in abdominal circumference, which may have led to dysfunction of core muscles, including the multifidus 


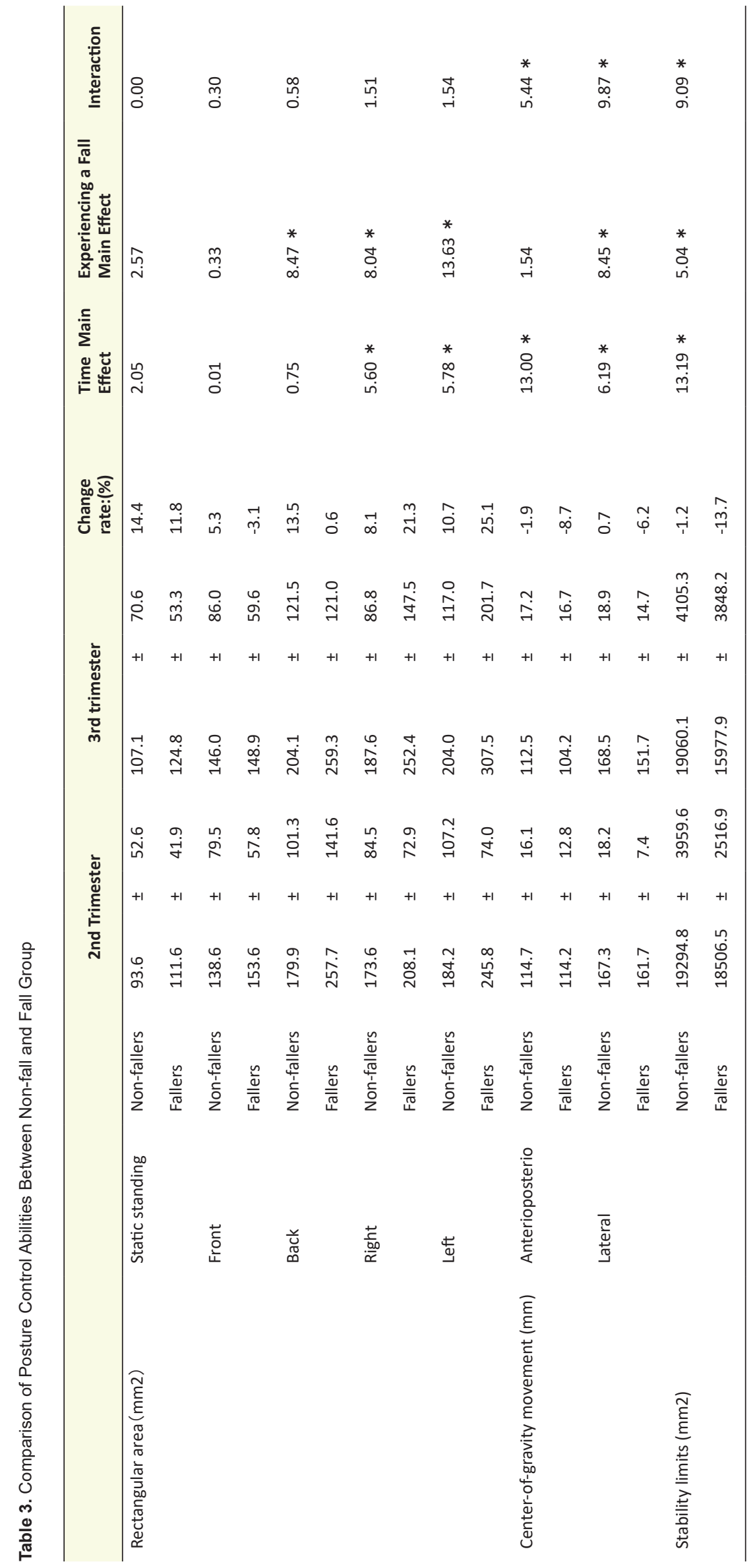


muscle, due to breakdown of the rectus abdominis muscle. Therefore, the lateral movement of the center of gravity decreased in the fall group due to the sudden increase in abdominal girth. Moreover, less movement of the center of gravity in the anteroposterior and lateral directions decreased the stability limits. A smaller stability limits mean there is a higher probability that the center of gravity line will deviate from the stable region, thereby lowering the stability of posture maintenance. Reduction of the stability limits associated with the course of pregnancy in the fall group may have influenced the occurrence of falls during pregnancy.

Rectangular areas in each direction except the front and during static standing were significantly increased. For the task in the present study, subjects were required to maintain their posture in the final region where center of gravity moved in each direction, except during the static upright position. The ankle flexors, hip extensors, joint dorsiflexors, hip flexors, and hip abductors must act together to maintain posture during forward movement. In the final region of the movement of the center of gravity in each direction, contraction of the muscles of the hip joint is required. The hip strategy involves control in the final area. Muscular strength during pregnancy is decreased in comparison with that before pregnancy $(18,19)$. The increase in rectangular area in each direction other than the front and during static standing was considered to be due to insufficient hip joint muscular strength relative to weight gain. It was thought that the invariance of the rectangular area at the time of static standing indicates a situation where balance is not required. The invariance of the front rectangular area was attributed to control using only the ankle joint strategy without using the hip joint strategy, as the amount of anteroposterior movement of the center of gravity was decreasing.

These results suggest that the postural control ability decreases temporally, but fluctuations at the time of movement of the center of gravity in the anteroposterior and lateral directions due to changes associated with pregnancy decrease the stability limits.

Changes in body weight and abdominal girth during the course of pregnancy seem to influence posture control, and while no significant differences in body weight were seen between the two groups at any time during pregnancy, abdominal girth was significantly smaller in the second trimester in the fall group. The effects of decreases in weight and abdominal girth on posture control from the second trimester in the fall group are thus complex. A check of medication history in the fall group revealed that one pregnant woman was taking medicine for anemia, so posture sway due to anemia from previous pregnancies may have been present. This suggests the possibility of original joint loosening or a decline in equilibrium function, due to insufficient weight control by muscle strength, in the fall group.

On the other hand, the rate of change in abdominal circumference from the second to third trimester was $9.9 \%$ in the fall group, significantly higher than the $6.9 \%$ increase in the non-fall group. Moreover, body weight gain was $6.4 \%$ in fallers, compared to $6.1 \%$ in nonfallers. Once possible factor related to falls is a rapid increase in abdominal girth over a short period of time, where the position of the center of gravity is rapidly displaced forward. Specifically, the substantial physical changes associated with pregnancy in a short period of time cause a shift in recognition of how the body moves $(20,21)$. In a study of 32 patients with chronic stroke hemiplegia, evaluation of the estimated reach distance of the nonparalyzed upper limb and examination of the relationship between error from actual reach distance and number of falls during hospitalization showed a significant correlation between error distance and the number of falls, with $92 \%$ of fallers reporting an error distance $\geq 6 \mathrm{~cm}$ $(21,22)$. In a previous study investigating the awareness of falls during pregnancy, more pregnant women reported they were not careful about falling compared to pregnant women who reported that they were careful about rising and squatting movements and falling while walking (3). Likewise, in the present study, a common response on the questionnaire survey from participants in the fall group was, "I fell down when I moved as usual", and shifts in how the body moves according to estimation errors may have been involved. The results of this study showed a decrease in posture control ability among fallers from before the second trimester. There was also a possibility of loosening of the joints and decreased equilibrium function from the second trimester, while abrupt increases in abdominal girth may have shifted recognition of how the body moved, i.e., estimation errors. Pregnant women need to consider not only incorporating exercises for posture control from before the second trimester into their daily routine but also utilizing information and guidance on fall prevention during pregnancy to manage the inevitable reductions in posture control ability observed from the second trimester. According to Whipple et al, the features of effective posture control training are: 1) body-weight exercises; 2) interactions between the body and head (eyeball movement), including quick movement in the horizontal direction; and 3) activation of muscle groups including amplitude motion in the vertical direction and the thigh and hip joint (23). As a constituent element of posture control training during pregnancy, we propose incorporating anti-gravity movements to promote muscle activity supporting bodyweight while being conscious of "wobble" in the front, rear, left and right directions due to weight shift. Consideration of these movements to facilitate recognition of displacement due to changes in weight and the body's center of gravity accompanying the progression of pregnancy is important.

One limitation of this study was that we could not capture changes in posture control before the second trimester, and so could not narrow down causes of decreases in posture 
control ability. The low number of participants who experienced falls is also a limitation. Future investigations should examine the effects of exercise on posture control ability along with changes in posture control from early pregnancy.

\section{Conflict of Interests}

Authors declare that they have no conflict of interests.

\section{Ethical Issues}

The ethical committee of Kansai University of Welfare Sciences approved

the study.

\section{Financial Support}

This research was conducted with the aid of a MEXT 2011 research grant (\#23593327).

\section{Acknowledgements}

The authors offer sincere thanks to the pregnant women who participated in the study, and the instructors in the Departments of Midwifery and Obstetrics and the gynecology clinic.

\section{References}

1. Dunning K, LemastersG, Levin L, et al. Falls in workers during pregnancy: Risk factors, job hazards, and high risk occupations. Am J Indusrial Med 2003; 44:664-72. doi:10.1002/ajim.10318

2. Connolly AM, Katz VL, Bash KL, McMahon MJ, Hansen WF. Truma and pregnancy. Am J Perinatol 1997;14(6):33136. doi:10.1055/s-2007-994155

3. Takeda K, Imura M. Survey of falls in pregnant women. Japanese Journal of Maternal Health. 2016;56(4):591-598.

4. Snijders C, Seroo J, Snijder J, Hoedt H. Change in form of the spine as a consequence of pregnancy. 11th international conference on Medical and Biological Engineering; Ottawa, Ontario, Canada; 1976:670-1.

5. Fast A, Weiss L, Ducommun EJ, Medina E, Butler JG. Low-back pain in pregnancy. Abdominal muscles, sit-up performance, and back pain. Spine 1990;15(1):28-30.

6. Calguneri $\mathrm{M}$, Bird HA, Wright V. Changes in joint laxity occurring during pregnancy. Ann Rheum Dis. 1982;41(2):126-8.

7. Jang J, Hsiao KT, Hsiao-Wecksler ET. Balance (perceived and actual) and preferred stance width during pregnancy. Clin Biomech. 2008;23:468-76. doi: 10.1016/j. clinbiomech.2007.11.011

8. Butler EE, Colon I, Druzin ML, Rose J. Postural equilibrium during pregnancy: Decreased stability with an increased reliance on visual cues. Am J Obstet Gynecol. 2006;195:1104-8. doi:10.1016/j.ajog.2006.06.015

9. Takeda K, Shimizu K, Imura M. Change of stability limits in the third trimester. J Phys Ther Sci. 2015;27(6):1813-1817. doi: $10.1589 /$ jpts.27.1813.

10. Shumway-Cook A, Woollacott MH. Motor Control. 3rd ed. Lippincott Williams \& Wilkins; 2007:158-186.

11. Mituko F, Midori W. Body measurement measurements of parts of the body (8) -pregnant and nursing women (1). Bulletin of Hiroshima Jogakuin University. 1971;21:75-191.

12. Wimalawansa SJ, Wikkramanayake TW. Factors affecting weight gain during Pregnancy and the Growth of the Infant. Ceylon J Med. 1987;30 (1):21-38.

13. Gibson MJ, Andres RO, Isaacs B, Radebaugh T, WormPetersen J. The prevention of falls in later life. A report of the Kellogg International work group on the prevention of falls by the elderly. Danish Medical Bulletin. 1987; 34(Suppl 4):1-24.

14. Mochizuki $\mathrm{H}$. The relationship between the perceived postural stability and indices of balance ability measured by posturography. Bunkyo Jounal of Health Science Techology. 2009; (2):55-60.

15. American Physical Therapy Association. Perinatal Exercise Guideline. Section on Obstetrics and Gynecology, Alexandria VA 1986.

16. Fries EC, Hellebrandt FA. The influence of pregnancy on the location of the center of gravity, postural stability, and body alignment. Am J Obstet Gynecol 1943;46:374-80.

17. Noguchi $S$, Tamaki $M$, Inoue $T$, Nakamichi $T$, Fujimoto M, Suzuki T. Muscle activity patterns of the multifidus, iliocostalis, and longissimus during lateral weight shift in the standing position. Journal of Kansai Physical Therapy. 2015; 15: 61-65.

18. Treuth MS, Butte NF, Puyau M. Pregnancy-related changes in physical activity, fitness and strength. Med Sci Sports Exerc. 2005;37:832-837. doi: 10.1249/01. MSS.0000161749.38453.02.

19. Geneviéve D, Charpentier K. Comparison of strength between pregnant and non-pregnant women. http://www. asbweb.org. May 16, 2016.

20. Okada Y, Takatori K, Nagino K, Tokumoto K, Ikeno K, Shomoto K. Relationship between Error in Estimated Reach Distance and Falls in Community-Dwelling Elderly People. The Journal of Japanese Physical Therapy Association. 2008;35(6):279-284.

21. Robinovitch SN, Cronin T. Perception of postural limits in elderly nursing home and day care participants. J Gerontol A Biol Sci Med Sci. 1999;54(3):B124-30.

22. Takatori K, Okada Y, Nagino K, et al. Relationship between Estimation Error of Perceived Reachability and the Number of Fall among Patients with Hemiparesis after Stroke. The Journal of Japanese Physical Therapy Association 2007;34(2): 52-58.

23. Whipple RH. Improving balance in older adults, Identifying the significant training stimuli. In: Masdeu JC, Sudarsky L, Wolfson L, eds. Gait Disorders of Aging Falls and Therapeutic Strategies. New York: Lippincott-Raven; 1997;355-379.

(C) 2018 The Author (s); This is an open-access article distributed under the terms of the Creative Commons Attribution License (http://creativecommons.org/licenses/by/4.0), which permits unrestricted use, distribution, and reproduction in any medium, provided the original work is properly cited. 